The following resources related to this article are available online at www.sciencemag.org (this information is current as of December 3, 2009 ):

Updated information and services, including high-resolution figures, can be found in the online version of this article at:

http://www.sciencemag.org/cgi/content/full/302/5648/1172

Supporting Online Material can be found at:

http://www.sciencemag.org/cgi/content/full/302/5648/1172/DC1

This article has been cited by 75 article(s) on the ISI Web of Science.

This article has been cited by 11 articles hosted by HighWire Press; see:

http://www.sciencemag.org/cgi/content/full/302/5648/1172\#otherarticles

This article appears in the following subject collections:

Science and Policy

http://www.sciencemag.org/cgi/collection/sci_policy

Information about obtaining reprints of this article or about obtaining permission to reproduce this article in whole or in part can be found at:

http://www.sciencemag.org/about/permissions.dtl 


\title{
Human Population: The Next Half Century
}

\author{
Joel E. Cohen
}

\begin{abstract}
By 2050, the human population will probably be larger by 2 to 4 billion people, more slowly growing (declining in the more developed regions), more urban, especially in less developed regions, and older than in the 20th century. Two major demographic uncertainties in the next 50 years concern international migration and the structure of families. Economies, nonhuman environments, and cultures (including values, religions, and politics) strongly influence demographic changes. Hence, human choices, individual and collective, will have demographic effects, intentional or otherwise.
\end{abstract}

It is a convenient but potentially dangerous fiction to treat population projections as exogenous inputs to economic, environmental, cultural, and political scenarios, as if population processes were autonomous. Belief in this fiction is encouraged by conventional population projections, which ignore food, water, housing, education, health, physical infrastructure, religion, values, institutions, laws, family structure, domestic and international order, and the physical and biological environment. Other biological species are recognized explicitly only in the recent innovation of quantifying the devastating demographic impacts of HIV and AIDS. The absence from population projection algorithms of influential external variables indicates scientific ignorance of how external variables influence demographic rates rather than any lack of influence (1).

Demographic projections stimulate fears of overpopulation in some, fears of demographic decline and cultural extinction in others (2). This review of current projections for the next half century will not attempt to assess the implications of likely demographic changes for health, nutrition, prosperity, international security, the physical, chemical and biological environment, or human values. Other articles in this series cover such topics.

\section{Past Population}

Earth's population grew about 10-fold from 600 million people in 1700 to 6.3 billion in 2003 (3). These and all demographic statistics are estimates; repeated qualifications of uncertainty will be omitted. It took from the beginning of time until about 1927 to put the first 2 billion people on the planet; less than 50 years to add the next 2 billion people (by 1974); and just 25 years to add the next 2 billion (by 1999). The population doubled in the most recent 40 years. Never before the second half of the 20th century had any person lived through a doubling of global population. Now some have lived through a tripling. The human species lacks

Rockefeller University and Columbia University, 1230 New York Avenue, Box 20, New York, NY 10021, USA. E-mail: cohen@rockefeller.edu any prior experience with such rapid growth and large numbers of its own species.

From 1750 to 1950, Europe and the New World experienced the most rapid population growth of any region, while the populations of most of Asia and Africa grew very slowly. Since 1950, rapid population growth shifted from Western countries to Africa, the Middle East, and Asia.

The most important demographic event in history occurred around 1965-70. The global population growth rate reached its all-time peak of about $2.1 \%$ per year ( $\mathrm{pa}$ ). It then gradually fell to $1.2 \%$ pa by 2002 (4). The global total fertility rate fell from 5 children per woman per lifetime in $1950-55$ to 2.7 children in $2000-05$. The absolute annual increase in population peaked around 1990 at 86 million and has fallen to 77 million. Concurrent trends included worldwide efforts to make contraception and reproductive health services available, improvements in the survival of infants and children, widespread economic development and integration, movements of women into the paid labor market, increases in primary and secondary education for boys and girls, and other cultural changes.

In 1960, five countries had total fertility rates at or below the level required to replace the population in the long run. By 2000, there were 64 countries such countries, with about $44 \%$ of all people $(4,5)$.

Worldwide urbanization has taken place for at least two centuries and accelerated greatly in the 20 th century. In 1800 , roughly $2 \%$ of people lived in cities; in 1900, $12 \%$; in 2000, more than $47 \%$, and nearly $10 \%$ of those city dwellers lived in cities of 10 million people or larger. Between 1800 and 1900 , the number of city dwellers rose more than 11-fold, from 18 million to 200 million; between 1900 and 2000, the number of city dwellers rose another 14-fold or more, from 200 million to 2.9 billion. In 1900, no cities had 10 million people or more. By 1950, one city did: New York. In 2000, 19 cities had 10 million people or more. Of those 19 cities, only four (Tokyo, Osaka, New York, and Los Angeles) were in industrialized countries (6).

\section{Demographic Projections of the Next 50 Years}

Projections of future global population prepared by the United Nations Population Division, the World Bank, the United States Census Bureau, and some research institutions assume business as usual (7-9). They include recurrent catastrophes to the extent that such catastrophes are reflected in past trends of vital rates, but exclude catastrophes of which there is no prior experience, such as thermonuclear holocaust or abrupt, severe climate change. The following summary relies mainly on the United Nations Population Division's urbanization forecasts (6) and World Population Prospects: The 2002 Revision (4). Alternative projections prepared by the UN include low, medium, high and constant-fertility variants. Estimates of present levels of demographic variables are projections based on measurements in recent years, rather than global current measurements.

According to the medium variant, the world's population is expected to grow from 6.3 billion today to 8.9 billion in 2050 . Whereas the first absolute increase by 1 billion people took from the beginning of time until about 1800 , the increase by one billion people from 6.3 billion to 7.3 billion is projected to require 13 to 14 years. The anticipated increase by 2050 of 2.6 billion over today's population exceeds the total population of the world in 1950, which was 2.5 billion.

Current absolute and relative global population growth rates are far higher than any experienced before World War II. The annual addition of 77 million people poses formidable challenges of food, housing, education, health, employment, political organization and public order. Virtually all of the increase is and will be in the economically less-developed regions. More than half of the annual increase currently occurs in six countries: India, China, Pakistan, Bangladesh, Nigeria, and the United States. Of the total annual increase, the United States accounts for $4 \%$.

Were fertility to remain at present levels, the population would grow to 12.8 billion by 2050 , more than double its present size. The medium projection of 8.9 billion people in 2050 assumes that efforts to make means of family planning available to women and couples will continue and will succeed, and that after 2010 high-risk behaviors related to AIDS will become less frequent and chances of infection among those engaging in high risk behaviors will decline. 


\section{Noviser}

The UN's 2002 estimate of 8.9 billion people in 2050 is 0.4 billion lower than that in their 2000 medium variant. About half of the decrease in the projection for 2050 is due to fewer projected births and about half to more projected deaths, notably from AIDS.

Global statistics conceal vastly different stories in different parts of the world. In 2000, about 1.2 billion people lived in the economically rich, more developed regions: Europe, Northern America, Australia, New Zealand, and Japan. The remaining 4.9 billion lived in the economically poor, less developed regions.

The current annual growth rate of global population is $1.22 \%$. Rich regions' population currently increases $0.25 \%$ annually. Poor regions' population grows $1.46 \%$ annually, nearly six times faster. The population of the least developed regions, the 49 countries where the world's poorest 670 million people lived in 2000 , annually increases $2.41 \%$. By 2050 , the projected annual growth rate of global population is $0.33 \%$. The poor countries' population will still be increasing $0.4 \%$ annually, whereas the population of the rich countries will have been declining for 20 years and will then be falling at $-0.14 \%$ annually. Thirty of the more developed countries are expected to have lower populations in 2050 than today, including Japan (14\% smaller), Italy (22\% smaller), and the Russian Federation (29\% smaller). By contrast, the population of today's poor countries is projected to rise to 7.7 billion in 2050 from 4.9 billion in 2000. Fertility in the less developed regions is expected to fall to replacement level in 2030-2035 but to remain above 2 children per woman by 2050 because some of the least developed countries will still have total fertility rates well above replacement level. The population of these high-fertility poor countries will be an increasing proportion of the population of the less developed regions.

The world's average population density of 45 people $/ \mathrm{km}^{2}$ in 2000 is projected to rise to 66 people $/ \mathrm{km}^{2}$ by 2050 . Globally, perhaps $10 \%$ of land is arable, so population densities per unit of arable land are roughly 10 times higher. In the rich countries, the population density was 23 people $/ \mathrm{km}^{2}$ in 2000 - half the global averageand was projected not to change at all by 2050 . In the poor countries, the population density was 59 people $/ \mathrm{km}^{2}$ in 2000 and was projected to rise to 93 people $/ \mathrm{km}^{2}$ in 2050 . For comparison, the population density of Liechtenstein was 204 people $/ \mathrm{km}^{2}$ in 2000 and that of the United States was 30. A population density of 93 people $/ \mathrm{km}^{2}$ over the entire developing world will pose unprecedented problems of land use and preservation.

According to these projections, the ratio of population density in the poor countries to that in the rich countries is projected to rise from 2.6 in 2000 to 4.0 in 2050 . Over the same interval, while the population density of Europe is projected to drop from 32 to 27 people $/ \mathrm{km}^{2}$, that of Africa is projected to rise from 26 to 60 people $/ \mathrm{km}^{2}$. The ratio of population density in Africa to that in Europe is projected to rise from 0.8 in 2000 to 2.2 in 2050. It seems plausible to anticipate increasing human effects on the natural environment in Africa and increasing pressure of migrants from Africa to Europe.

The difference in population growth rate between rich and poor countries affects both population size and age structure. If a population grows slowly, the number of births each year nearly balances the number of deaths. As most deaths occur at older ages, the numbers of individuals in different age groups are roughly equal up to older ages. The so-called population pyramid of a slowly growing population resembles a column (Fig. 1, middle row left) (10). If a population grows rapidly, each birth cohort is larger than its predecessor and the population pyramid is triangular (Fig. 1, middle row right). The projected difference in age structures between the European Union versus North Africa and western Asia (Fig. 1, bottom) has obvious implications for the supplies of military personnel and ratios of elderly to middle-aged.

Inequality in the face of death between rich and poor will decrease but remain large if survival improves everywhere as anticipated in the coming half century. Global life expectancy in $2000-05$ is estimated at 65 years; in 2045-50, at 74 years. Over the same interval, life expectancy in the rich countries is expected to rise from 76 years to 82 years and in the poor countries from 63 years to 73 years. The average infant born in a poor country had a chance of dying before age 1 that was 8.1 times higher than that in a rich country in 2000-05; the same ratio is projected to be 5.2 in 2045-50.

Despite higher death rates, poor countries' populations grow faster than those of rich because birth rates in poor countries are much higher. At current birth rates, during her lifetime, the average woman in the poor countries bears nearly twice as many children (2.9) as in the rich countries (1.6). By 2050, according to the medium variant, the total fertility rate in today's poor countries will drop to 2.0. The total fertility rate in today's more developed countries is projected to rise to almost 1.9 children per woman, as timing effects that currently depress the total fertility rate cease to operate.

In the coming decade, more than half of all people will live in cities, for the first time in human history. Almost all population growth in the next half century will be in cities in poor countries while the world's rural population will remain flat, near 3 billion people.
The United Nations Population Division projects urban population only as far as 2030 (6). Its figures on urbanization disguise major ambiguities and variations among countries in definitions of "cities" and "urban." Nevertheless, the trend toward urbanization is clear. Of the projected 2.2-billion increase in population from 2000 to $2030,2.1$ billion will be in urban areas, and all but 0.1 billion of that urban increase will be in developing countries. The annual rate of increase of urban population over the next 30 years, $1.8 \%$, is nearly twice the projected annual rate of increase of global population during that period. The urban population of developing regions will grow rapidly as people migrate from rural to existing urban areas and transform rural settlements into cities. The rural population of the rich countries peaked around 1950 and has slowly declined since then. The rural population of the presently poor countries is expected to peak around 2025 and then gradually decline. Urbanization of the rich countries will continue, rising from $75 \%$ of people in 2000 to $83 \%$ in 2030 . Over the same period, urbanization of the poor countries will rise from $40 \%$ to $56 \%$, similar to the level of urbanization in the rich countries in 1950.

The coming half century will see dramatic population aging, which means a higher proportion of the population in elderly age groups. The proportion of children aged 4 years and under peaked in 1955 at $14.5 \%$ and gradually declined to $10.2 \%$ in 2000 . By contrast, the fraction of people aged 60 years and older gradually increased from a low of $8.1 \%$ in 1960 to $10.0 \%$ in 2000 . Each group constitutes about $10 \%$ of humanity today. The 20th century will probably be the last in which younger people outnumbered older ones. Children aged 0 to 4 are projected to decline to $6.6 \%$ of global population by 2050 , whereas people aged 60 years and older are projected to more than double to $21.4 \%$. By 2050 , there will be 3.2 people aged 60 years or older for every child 4 years old or younger. This reversal in the numerical dominances of old and young reflects improved survival and reduced fertility. Improved survival raised the global average length of life from perhaps 30 years at the beginning of the 20th century to 65 years at the beginning of the 21st. Reduced fertility rates added smaller cohorts to the younger age groups.

Because the populations of the poor countries have been growing more rapidly than those of the rich, they have a much higher fraction of people under the age of 15 years (33\% versus $18 \%$ in 2000). By 2050, in the medium variant, these fractions will drop to $21 \%$ and $16 \%$ in poor and rich countries, respectively. The global 


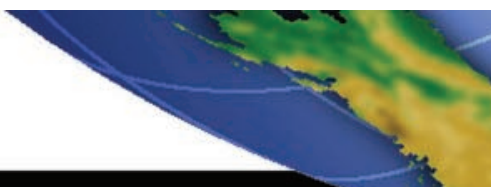

State of the Planet

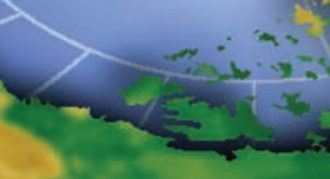

$--$

14 Nov. - 5 Dec. 2003

fraction of the elderly population (aged 65 years or more) will rise from $7 \%$ in 2000 to $16 \%$ by 2050 . Over the same period, the elderly fraction will rise from 5 to $14 \%$ in the presently poor countries and from 14 to $26 \%$ in the rich countries. Though the fraction of children in the population will decrease by more in the poor countries than in the rich, the fraction of elderly will increase by more in the rich countries than in the poor. Both shifts will have consequences for spending on the young and the old.
Slowly growing populations have a higher elderly dependency ratio (the ratio of the number of people aged 65 and older to the number aged 15 to 64 ), while rapidly growing populations have a higher youth dependency ratio (the ratio of the number of people aged 0 to 14 to the number aged 15 to 64 ). The elderly dependency ratio rose from 1950 to 2000 at a rapid rate in the more developed countries, slightly less rapidly in the United States, and still less rapidly in the world as a
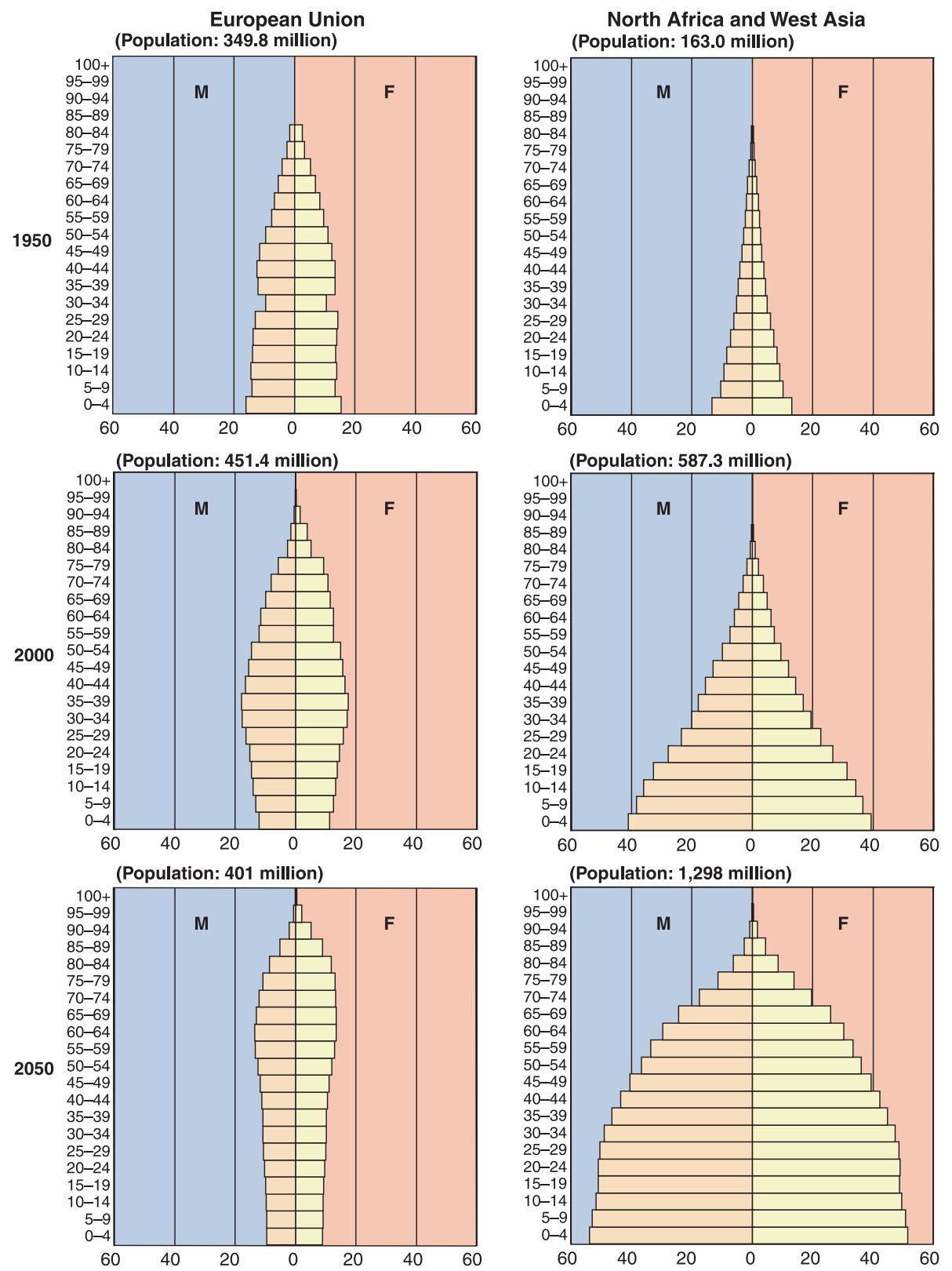

Fig. 1. Population size and age distribution for 1950, 2000, and 2050 in an anticipated enlarged European Union of 25 countries and in 25 countries of North Africa and West Asia between India's western border and the Atlantic Ocean, excluding countries of central Asia that were part of the former Soviet Union, those of Muslim black Africa, and Israel (10). Horizontal scale gives million persons separately by sex; vertical scale gives age groups in increments of 5 years.

whole. The ratio rose only slightly in the less developed countries, and hardly at all in the least developed countries. After 2010, in the more developed countries, the United States, and the less developed countries, the elderly dependency ratio will increase sharply faster; this acceleration will be greater in the more developed countries and the United States. The least developed countries will experience a slow increase in the elderly dependency ratio after 2020 and, by 2050, will be approaching the elderly dependency ratio of the more developed countries in 1950 .

\section{Demographic Uncertainties: Migration and the Family}

According to the United Nations Population Division, "International migration is the component of population dynamics most difficult to project reliably. This occurs in part because the data available on past trends are sparse and partial, and in part because the movement of people across international boundaries, which is a response to rapidly changing economic, geopolitical or security factors, is subject to a great deal of volatility" (11). The UN's 2002 medium variant posits migration from less to more developed regions of 2.6 million people annually during 1995-2000, declining to nearly 2.0 million by $2025-30$, and remaining constant at that level until 2050. The United States is anticipated to increase annually by 1.1 million of these 2 million migrants, more than five times the number expected to be added annually to the next largest recipient, Germany $(211,000)$. The major sending countries are expected to be China, Mexico, India, the Philippines, and Indonesia.

International migration is likely to remain important for specific countries, including the United States. In the mid-1990s, about 125 million people ( $2 \%$ of world population) resided outside of their country of birth or citizenship. In 1990 , only 11 countries in the world had more than 2 million migrants, and they collectively had almost 70 million migrants. The largest numbers of migrants were in the United States (19.6 million), India (8.7 million), Pakistan (7.3 million), France (5.9 million), and Germany (5.0 million). The countries with the highest percentage of international migrants in the total population were countries with relatively small populations. In the United Arab Emirates, Andorra, Kuwait, Monaco, and Qatar, 64 to $90 \%$ of the population were immigrants.

If predicting international migration is difficult, predicting change in family structure is more difficult. Goldscheider (12) suggested that the fall in fertility during the demographic transition weakened the ties between men 


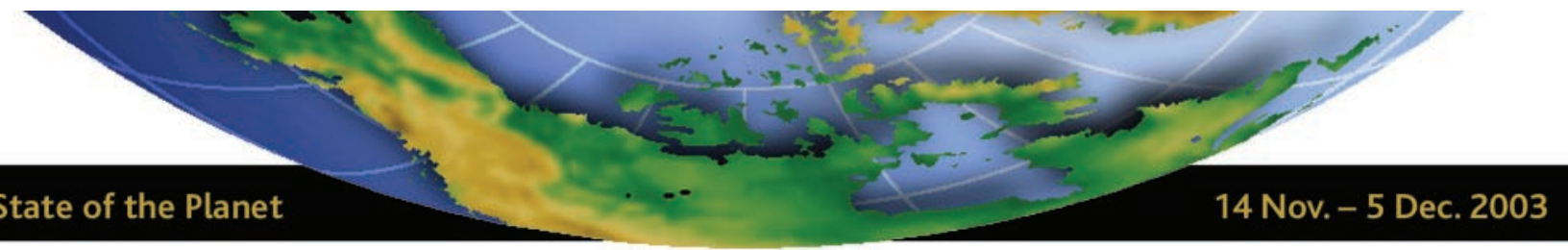

and women based on parenthood and that the rise in divorce and cohabitation is weakening the ties between fathers and children. Nonmarital births increased as a percentage of all births in the United States from 5.3\% in 1960 to $33.0 \%$ in 1999 . In 1999 , the United States had 1.3 million births to unmarried women (13). In 1998, Iceland, Norway, Sweden, Denmark, France, United Kingdom, and Finland all had higher proportions of nonmarital births than the United States. By contrast, in Germany, Italy, Greece, and Japan, less than $15 \%$ of births were nonmarital (13). Among United States women aged 15 to 29 years at first birth, when that first birth was conceived before marriage, the fraction who married before the birth fell from $60 \%$ in $1960-64$ to $23 \%$ in 1990-94 (14). By 1994, about $40 \%$ of children in the United States did not live with their biological father (12).

In the United States, the number of widowed males aged 55 to 64 per thousand married persons fell from 149 in 1900 to 35 in 2000, whereas the number of divorced males aged 55 to 64 per thousand married persons rose from 7 to 129 . Divorced males became more frequent than widowed males between 1970 and 1980. Divorced females became more frequent than widowed females between 1990 and 2000. By 2000, the number of divorced and widowed persons aged 55 to 64 per thousand married persons was 164 males and 426 females (2.6 such females for each such male) (15). Remarriages and stepfamilies are becoming increasingly common.

Three factors set the stage for further major changes in families: fertility falling to very low levels; increasing longevity; and changing mores of marriage, cohabitation, and divorce. In a population with one child per family, no children have siblings. In the next generation, the children of those children have no cousins, aunts, or uncles. If adults live 80 years and bear children between age 20 and 30 on average, then the parents will have decades of life after their children have reached adulthood and their children will have decades of life with elderly parents. The full effects on marriage, child bearing, and child rearing of greater equality between the sexes in education; earnings; and social, legal, and political rights have yet to be felt or understood.

\section{References and Notes}

1. J. E. Cohen, How Many People Can the Earth Support? (W. W. Norton, New York, 1995).

2. L. Shriver, Popul. Dev. Rev. 29 (no. 2), 153 (2003).

3. United States Census Bureau, Historical Estimates of World Population (online). Available at www. census.gov/ipc/www/worldhis.html (cited 21 June 2003).

4. United Nations Population Division, World Population Prospects: the 2002 Revision, Highlights (online database). ESA/P/WP.180, revised 26 February 2003, p. vi. Available at: http://esa.un.org/unpp/ (consulted 1 to 30 June 2003)

5. United Nations Population Division, Partnership and Reproductive Behaviour in Low-Fertility Countries,
ESA/P/WP.177, revised May 2003. Available at www. un.org/esa/population/publications/reprobehavior/ partrepro.pdf (cited 29 June 2003).

6. United Nations Population Division, World Urbanization Prospects: The 2001 Revision. ESA/P/WP.173. (United Nations, New York, 2002).

7. J. Bongaarts, R. A. Bulatao, Eds. Beyond Six Billion: Forecasting the World's Population (National Academy Press, Washington, DC, 2002).

8. J. E. Cohen, in Seismic Shifts: the Economic Impact of Demographic Change, J. S. Little, R. K. Triest, Eds., Federal Reserve Bank of Boston, conference series no. 46, 11 to 13 June 2001 (Federal Reserve Bank of Boston, Boston, MA, 2001), pp. 83-113.

9. J. E. Cohen, in What the Future Holds: Insights from Social Science, R. N. Cooper, R. Layard, Eds. (MIT Press, Cambridge, MA, 2002), pp. 29-75.

10. P. Demeny, Popul. Dev. Rev. 29 (no. 1), 1 (2003).

11. United Nations Population Division, World Population Prospects: The 1998 Revision: Volume III: Analytical Report. ESA/P/WP.156, revised 18 November 1999 (United Nations, New York, 1999).

12. F. K. Goldscheider, Futurist 32, 527 (2000).

13. S. J. Ventura, C. A. Bachrach, National Vital Statistics Reports 48 (no. 16, revised), 18 October 2000. Available at www.cdc.gov/nchs/data/nvsr/nvsr48/nvs48 16.pdf (cited 25 June 2003).

14. A. Bachu, Current Population Reports; P23-197 (U.S. Census Bureau, Washington, DC, 1999).

15. P. Uhlenberg, in United Nations, Department for Economic and Social Information and Policy Analysis, 1994. Ageing and the Family. Proceedings of the United Nations International Conference on Ageing Populations in the Context of the Family, ST/ESA/SER.R/124 (United Nations, New York, 1994), pp. 121-127.

16. I acknowledge with thanks the support of U.S. National Science Foundation grant DEB 9981552, the assistance of K. Rogerson, and the hospitality of Mr. and Mrs. W. T. Golden during this work.

\section{Web Resources}

www.sciencemag.org/cgi/content/full/302/5648/1172/ DC2

\title{
Prospects for Biodiversity
}

\author{
Martin Jenkins
}

\begin{abstract}
Assuming no radical transformation in human behavior, we can expect important changes in biodiversity and ecosystem services by 2050. A considerable number of species extinctions will have taken place. Existing large blocks of tropical forest will be much reduced and fragmented, but temperate forests and some tropical forests will be stable or increasing in area, although the latter will be biotically impoverished. Marine ecosystems will be very different from today's, with few large marine predators, and freshwater biodiversity will be severely reduced almost everywhere. These changes will not, in themselves, threaten the survival of humans as a species.
\end{abstract}

What will be the state of the world's biodiversity in 2050, and what goods and services can we hope to derive from it? First, some assumptions: that the United Nations median population estimate for 2050 holds, so that Earth will have roughly nine billion people-just under half again as many as

United Nations Environment Programme-World Conservation Monitoring Centre, 219c Huntingdon Road, Cambridge, CB3 ODL, UK. E-mail: martin. jenkins@unep-wcmc.org are currently alive $(1,2)$; that the Intergovernmental Panel on Climate Change scenarios provide a good indication of global average surface temperatures and atmospheric $\mathrm{CO}_{2}$ concentrations at that time, with the former $\sim 1^{\circ} \mathrm{C}$ to $2^{\circ} \mathrm{C}$ and the latter $\sim 100$ to 200 parts per million higher than today (3); and, perhaps most important, although most nebulous, that humanity as a whole has not determined on a radically new way of conducting its affairs. Here, then, is a plausible future.
In this future, the factors that are most directly implicated in changes in biodiversity-habitat conversion, exploitation of wild resources, and the impacts of introduced species (4) -will continue to exert major influences, although their relative importance will vary regionally and across biomes. In combination, they will ensure continuing global biodiversity loss, as expressed through declines in populations of wild species and reduction in area of wild habitats.

\section{Extinction Rates}

To start, as it were, at the end: with extinction, perhaps the most tangible measure of biodiversity loss. The uncertainties that still surround our knowledge of tropical biotas (which include the great majority of extant species); the difficulty of recording extinctions; and our ability, when we put our minds to it, to bring species back from the brink 